Cartas científicas

Rev Urug Cardiol 2019; 34:218-222

doi: 10.29277/cardio.34.2.17

\title{
Preeclampsia como desencadenante de crisis tirotóxica: reporte de caso
}

\section{Resumen}

La crisis tirotóxica o tormenta tiroidea desencadenada por preeclampsia es poco frecuente. Es importante llegar a un diagnóstico y tratamiento precoces, dado que su retraso conlleva alta morbimortalidad para el binomio materno-fetal.

Reportamos el caso de una paciente cursando tercer trimestre de gestación que instala una crisis tirotóxica desencadenada por una preeclampsia. Se realiza interrupción del embarazo y tratamiento específico del factor precipitante y de la tormenta tiroidea, con buena evolución posterior.

Palabras clave: $\quad$ PREECLAMPSIA

CRISIS TIROIDEA

HIPERTIROIDISMO

Preeclampsia as a trigger of thyrotoxic crisis: a case report

\section{Summary}

Thyrotoxic crisis or thyroid storm is rarely triggered by preeclampsia. It is important to reach early diagnosis and treatment, since its delay leads to high morbidity and mortality for the maternal-fetal binomial. We hereby report the case of a patient that develops a thyroid storm triggered by severe preeclampsia during the third trimester of pregnancy. Pregnancy interruption and specific treatment of the precipitating factor and the thyroid storm are performed, with good subsequent progress.

Key words: $\quad$ PRE-ECLAMPSIA

THYROID CRISIS

HYPERTHYROIDISM

Pré-eclâmpsia como desencadeador de crise tireotóxica: relato de caso

\section{Resumo}

A crise tireotóxica ou tempestade tireoidiana desencadeada pela pré-eclâmpsia é rara. É importante o diagnóstico e tratamento precoces, pois o atraso leva a alta morbimortalidade para o binômio materno-fetal. Relatamos o caso de uma paciente com hipertireoidismo que está no terceiro trimestre de gravidez e instala uma tempestade tireoidiana desencadeada por pré-eclâmpsia grave. Interrupção da gravidez e tratamento específico do fator precipitante e da tempestade tireoidiana são realizados, com boa evolução posterior.

Palavras-chave: PRE-ECLÂMPSIA

CRISE TIRÓIDEA

HIPERTIREOIDISMO

Sra. Editora

\section{Introducción}

El hipertiroidismo durante el embarazo multiplica por cinco el riesgo de preeclampsia y por diez el riesgo de crisis tirotóxica(1).
La preeclampsia es la aparición de hipertensión arterial (presión arterial $[\mathrm{PA}] \geq 140 / 90 \mathrm{mmHg}$ en dos tomas separadas por 4 a 6 horas) y proteinuria, o hipertensión y disfunción orgánica (con o sin proteinuria) después de las 20 semanas de gestación ${ }^{(2)}$.

La crisis tirotóxica o tormenta tiroidea es una emergencia endocrinológica. Constituye una complicación hipermetabólica, potencialmente fatal,

Recibido May 12, 2019; aceptado Jun 4, 2019. 
Tabla 1. Criterios diagnósticos de la crisis tirotóxica (escala de Burch y Wartofsky).

Parámetros diagnósticos

Puntos

Disfunción termorreguladora (temperatura axilar)

$\begin{array}{lr}37,2-37,7{ }^{\circ} \mathrm{C} & 5 \\ 37,8-38,2{ }^{\circ} \mathrm{C} & 10 \\ 38,3-38,8{ }^{\circ} \mathrm{C} & 15 \\ 38,9-39,3{ }^{\circ} \mathrm{C} & 20 \\ 39,4-39,9{ }^{\circ} \mathrm{C} & 25 \\ >39,9{ }^{\circ} \mathrm{C} & 30\end{array}$

Efectos sobre el sistema nervioso central

Ausente

Leve (agitación)

Moderado (delirio, psicosis,

letargo marcado)

Severo (convulsiones, coma) 30

\section{Disfunción gastrointestinal-hepática}

Ausente

Moderada (diarrea, náuseas/vómitos, dolor abdominal)

Severa (ictericia inexplicable)

\section{Disfunción cardiovascular}

Taquicardia (latidos/minuto)

100-109 5

110-119

120-129

130-139

$>140$

25

\section{Insuficiencia cardíaca congestiva}

Ausente

Leve (edema de los pies)

Moderada (estertores crepitantes bibasales)

Severa (edema pulmonar)

Fibrilación auricular

Ausente
Presente

Evento precipitante

Ausente 0

Presente 10

Sistema de puntuación: una puntuación de 45 o más es altamente sugestiva de tormenta tiroidea; entre 25 y 44 puntos es sugestiva de tormenta inminente, y si es inferior a 25 es poco probable que se trate de esta entidad. Adaptado de Burch y Wartofsky, 1993. caracterizada por compromiso cardiovascular (taquicardia, arritmias, insuficiencia cardíaca), hipertermia y alteraciones neurológicas (irritabilidad, inquietud, confusión, delirio, convulsiones).

Puede estar presente en otras patologías como sepsis, feocromocitoma o hipertermia maligna, por lo que inicialmente el diagnóstico puede generar dificultades ${ }^{(1)}$.

La tormenta tiroidea es una exacerbación aguda de un hipertiroidismo que surge por la liberación de hormonas tiroideas a la circulación sistémica. Puede desencadenarse por diferentes factores ${ }^{(2)}$.

Cuando existe sospecha, el tratamiento no debe retrasarse pues es potencialmente mortal. El paciente debe ser monitorizado en cuidados intensivos, recibir tratamiento específico y eliminarse el evento precipitante en forma precoz ${ }^{(3)}$.

Es una patología poco frecuente, con una incidencia de 0,2 casos por 100.000 habitantes y tasa de mortalidad elevada, entre $10 \%$ y $30 \%{ }^{(4)}$. Se estima que la tormenta tiroidea ocurre en $1 \%$ a $2 \%$ de los embarazos en mujeres con hipertiroidismo.

El diagnóstico y tratamiento tempranos pueden descender la tasa de mortalidad a $10 \%{ }^{(5)}$, mientras que un diagnóstico tardío conlleva la posibilidad de shock o coma.

Los criterios clínicos más utilizados para el diagnóstico temprano de crisis tirotóxica son los de Burch y Wartofsky ${ }^{(6)}$, quienes plantearon una escala basada en el grado de disfunción multisistémica (tabla 1). Cuando la puntuación es $\geq 45$, es altamente sugestiva de tormenta tiroidea.

El perfil de laboratorio revela leucocitosis y enzimas hepáticas elevadas y ocasionalmente hipercalcemia.

Los resultados de las pruebas de función tiroidea son consistentes con hipertiroidismo (elevación de T4 libre/T3 libre y TSH deprimida), pero no siempre se correlacionan con la severidad de la tormenta tiroidea.

Es importante investigar factores precipitantes (tabla 2) para realizar tratamiento específico.

El diagnóstico de crisis tirotóxica puede ser difícil; la demora en el inicio del tratamiento adecuado conlleva alto riesgo para el binomio materno-fetal. Presentamos el caso clínico de una embarazada con crisis tirotóxica desencadenada por una preeclampsia.

\section{Caso clínico}

Paciente de 31 años, sexo femenino, con antecedentes personales de asma leve y ginecoobstétricos de dos gestas y dos partos vaginales, cursando gestación de 30 semanas irregularmente contro- 
Tabla 2. Precipitantes comunes de la tormenta tiroidea.

- Emergencia quirúrgica aguda

- Inducción de anestesia

- Cetoacidosis diabética

- Embolismo pulmonar

- Incumplimiento de terapia antitiroidea

- Infarto agudo de miocardio

- Infección

- Hipertensión/preeclampsia

- Trabajo de parto y parto

- Anemia severa

- Terapia con $\mathrm{I}^{131}$

lada. Consulta en emergencia por palpitaciones frecuentes, insomnio y labilidad emocional. Niega mareos, síncope, dolor precordial y disnea.

Examen: lúcida, eupneica, normocoloreada, bien hidratada y perfundida. Cardiovascular: ritmo regular de $100 \mathrm{cpm}$. No ruidos patológicos. $\mathrm{PA}=140 / 90 \mathrm{mmHg}$.

Pleuropulmonar: eupneica, murmullo alvéolo-vesicular conservado, sin estertores.

Electrocardiograma: ritmo sinusal de $100 \mathrm{cpm}$, Py PR normales, sin elementos de isquemia aguda.

Ya ingresada, presenta excitación psicomotriz, episodios de hipertermia (temperatura axilar $38^{\circ} \mathrm{C}$ ), taquicardia regular de 110 cpm e hipertensión arterial $(150 / 90 \mathrm{mmHg})$, iniciándose tratamiento con alfametildopa $500 \mathrm{mg}$ cada 12 horas.

A las 48 horas del ingreso presenta episodio de disnea sibilante de inicio brusco, con estertores secos difusos, por lo que se indican broncodilatadores. Concomitantemente dolor abdominal tipo cólico, difuso, a predominio en hipogastrio y náuseas; no vómitos. A nivel ginecológico, episodios de genitorragia escasa, sin repercusión hemodinámica ni hematimétrica. Valorada por ginecólogo, diagnostica placenta previa. No elementos de sufrimiento fetal.

Se solicita ingreso a la unidad cardiológica para monitorización y tratamiento. Al ingreso se constata paciente lúcida, inquieta, tolera el decúbito, exoftalmos bilateral, ventilando al aire, ritmo regular de $110 \mathrm{cpm}, \mathrm{PA}=160 / 100 \mathrm{~mm} \mathrm{Hg}$.

Abdomen: indoloro, altura uterina acorde a edad gestacional.

Sistema nervioso central: sin déficit neurológico.
De la paraclínica solicitada se destaca:

- Perfil tiroideo: TSH $=0,01 \mu \mathrm{U} / \mathrm{ml}$ (rango normal en el tercer trimestre entre 2,01 y 4,30 $\mu \mathrm{U} / \mathrm{ml}$ ). $\mathrm{T} 4=2,2 \mathrm{ng} / \mathrm{dl}$ (rango normal en el tercer trimestre entre 0,40 y $0,82 \mathrm{ng} / \mathrm{dl}$ ).

- Índice proteinuria/creatininuria $=0,7 \mathrm{mg} / \mathrm{mmol}$.

- Leucocitosis $=14.000 / \mathrm{mm}^{3}$.

Ante una embarazada con cifras de PA sistólica de $160 \mathrm{mmHg}$ e índice proteinuria/creatininuria > 0,3 $\mathrm{mg} / \mathrm{mmol}$, se realiza diagnóstico de preeclampsia severa.

Dado el perfil tiroideo, con TSH suprimida y niveles elevados de T4, se diagnostica hipertiroidismo.

Por presentar escala de Burch-Wartofsky de 50 puntos, dados por temperatura axilar $38^{\circ} \mathrm{C}=10$ puntos (p), taquicardia de $110 \mathrm{cpm}=10 \mathrm{p}$, dolor abdominal $=10 \mathrm{p}$, excitación psicomotriz $=10 \mathrm{p}$, con preeclampsia como factor precipitante $=10 \mathrm{p}$, se confirma el diagnóstico de crisis tirotóxica o tormenta tiroidea.

Ante el diagnóstico de crisis tirotóxica desencadenada por preeclampsia se decide interrupción del embarazo por cesárea, realizando previamente tratamiento de maduración fetal con dexametasona 6 mg cada 12 horas intramuscular.

Se indica terapia antitiroidea con metimazol a dosis de $1,5 \mathrm{mg} / \mathrm{kg} /$ día vía oral, bisoprolol $5 \mathrm{mg}$ vía oral cada 24 horas para controlar la frecuencia cardíaca a través del bloqueo de la unión de las catecolaminas a los receptores betaadrenérgicos y reducción de la conversión periférica de T4 a T3. Con este mismo objetivo se continúa el tratamiento con glucocorticoides: hidrocortisona con dosis carga de 300 $\mathrm{mg}$ intravenosa y mantenimiento con $50 \mathrm{mg}$ cada 8 horas.

La cesárea se lleva a cabo de urgencia sin incidentes, obteniéndose recién nacido vivo. Se realiza sulfato de magnesio con dosis carga de $4 \mathrm{~g}$, más dosis de mantenimiento de 24 g por bomba de infusión continua hasta las 24 horas siguientes a la cesárea, con el fin de prevenir la eclampsia.

Evoluciona lúcida, en normotermia, sin déficit neurológico focal. Ritmo regular de $90 \mathrm{cpm}$, PA 110/80 mmHg. Mejoría del broncoespasmo con broncodilatadores; saturación de oxígeno $99 \%$ ventilando al aire. Ecocardiograma sin alteraciones, fracción de eyección de ventrículo izquierdo: $70 \%$.

Sin actividad infecciosa. Urocultivo y hemocultivos negativos. Útero contraído y retraído, cicatriz quirúrgica sin elementos fluxivos.

Dada la estabilidad clínica, se decide alta a sala de obstetricia al tercer día de su ingreso a la unidad cardiológica. 


\section{Discusión}

La paciente presentó una crisis tirotóxica o tormenta tiroidea desencadenada por preeclampsia. Presentó 50 puntos de la escala de Burch y Wartofsky, que considera el diagnóstico de crisis tirotóxica cuando la puntuación es $\geq 45 \mathrm{p}$. Una vez confirmado el diagnóstico se realizó el tratamiento precozmente, y dado el cuadro de preeclampsia severa se interrumpió el embarazo mediante cesárea.

El cuadro clínico de crisis tirotóxica no está relacionado necesariamente con el nivel de hormonas tiroideas, pues los pacientes con tormenta tiroidea tienen mayor cantidad de receptores de unión a las catecolaminas que los pacientes con hipertiroidismo que no la desarrollan ${ }^{(5)}$.

El diagnóstico es clínico, ya que la paraclínica no evidencia diferencias claras con los pacientes con hipertiroidismo.

La alteración mental fue la única característica significativa que aparece al diferenciar tormenta tiroidea con hipertiroidismo no controlado.

En un estudio de cohorte de TE Angell y colaboradores, en Estados Unidos en 2015, la alteración de la vigilia se relacionó con mayor mortalidad y estadía en cuidados intensivos, de tal forma que la disfunción del sistema nervioso central se beneficia del tratamiento precoz y agresivo(6).

El tratamiento consiste en reposo, monitorización continua de PA y electrocardiograma en una unidad de cuidados cardiológicos intensivos.

Se debe reponer con cristaloides, ya que por la presencia de hipertermia e hipermetabolismo puede presentarse deshidratación ${ }^{(1)}$.

Para controlar la hipertemia se pueden realizar medidas físicas y paracetamol, evitando el ácido acetilsalicílico, ya que compite por la unión de las proteínas transportadoras de las hormonas tiroideas, pudiendo aumentar su concentración libre en sangre y empeorar el cuadro clínico.

Los betabloqueantes utilizados, como el bisoprolol, controlan la PA y la frecuencia cardíaca, y re- ducen la conversión periférica de T4 a T3; además, el bisoprolol tiene actividad cardioselectiva $\beta 1 \mathrm{y}$ menor aumento de la resistencia de la vía aérea que otros agentes en una paciente asmática.

Para bloquear la síntesis de hormona tiroidea se utilizó metimazol. Durante el primer trimestre del embarazo se recomienda el propiltiouracilo como antitiroideo, ya que su uso no está asociado a teratogenicidad, como el metimazol. En el segundo y tercer trimestres se opta por metimazol, dado que al propiltiuracilo puede asociarse en esta etapa a hepatotoxicidad con falla hepática fulminante ${ }^{(3)}$.

Los glucocorticoides son útiles para bloquear la conversión de T4 a T3 y como profilaxis de la insuficiencia adrenal relativa que se da concomitantemente con la tirotoxicosis. En este caso se usó hidrocortisona; como alternativa puede utilizarse dexametasona $2 \mathrm{mg}$ intravenosa cada 6 horas.

El tratamiento antitiroideo se debe continuar luego del parto, durante la lactancia, pudiéndose descender la dosis de metimazol a la $\operatorname{mitad}^{(2)}$.

\section{Conclusión}

El hipertiroidismo puede estar presente durante el embarazo, con una prevalencia entre $0,1 \%$ y $0,4 \%$.

El 1\% de las pacientes gestantes con hipertiroidismo puede progresar a una crisis tirotóxica. Aun con tratamiento agresivo la mortalidad es elevada, pudiendo llegar a 20\%. Existen pocos casos reportados de crisis tirotóxica precipitada por preeclampsia, trabajo de parto o cesárea.

El diagnóstico es clínico y debe realizarse precozmente junto a un tratamiento adecuado para evitar graves consecuencias al binomio madre-hijo.

Al identificar y tratar los factores desencadenantes también es posible disminuir la morbimortalidad materno-fetal.

Dres. Jorge Castelli, Anabela Fernández. Unidad Docente Asistencial de prevención, diagnóstico y tratamiento de patologías graves y potencialmente graves en obstetricia. Unidad Cardiológica. Hospital Militar. Montevideo, Uruguay

Correspondencia: Dra. Anabela Fernández.Correo electrónico: anabelafm@telefonica.net Los autores declaran no tener conflictos de intereses.

Jorge Castelli, https://orcid.org/0000-0002-9086-4915 Anabela Fernández, https://orcid.org/0000-0001-8362-1846 


\section{Bibliografía}

1. Parasa M, Chinthakunta BK, Vemuri NN, Shaik MS. Out of the blue! Thyroidcrisis. Anesth Essays Res. 2015; 9(1):130-2. doi: 10.4103/0259-1162. 150179

2. ACOG Committee on Practice Bulletins-Obstetrics. ACOG practice bulletin. Diagnosis and management of preeclampsia and eclampsia. Number 33, January 2002. Obstet Gynecol. 2002; 99(1): 159-67.

3. Bahn Chair RS, Burch HB, Cooper DS, Garber JR, Greenlee MC, Klein I, et al. Hyperthyroidism and other causes of thyrotoxicosis: management guidelines of the American Thyroid Association and American Association of Clinical Endocrinolo- gists. Thyroid 2011; 21(6): 593-646. doi: 10.1089/ thy. 2010.0417

4. Carroll R, Matfin G. Endocrine and metabolic emergencies: thyroid storm. Ther Adv Endocrinol Metab. 2010;1(3):139-45. doi: 10.1177/2042018810382481

5. Rashid M, Rashid MH. Obstetric management of thyroid disease. Obstet Gynecol Surv. 2007;62(10): 680-8. doi:10.1097/01.ogx.0000281558.59184.b5

6. Angell TE, Lechner MG, Nguyen CT, Salvato VL, Nicoloff JT, LoPresti JS. Clinical features and hospital outcomes in thyroid storm: a retrospective cohort study. J Clin Endocrinol Metab. 2015; 100(2):451-9. doi:10.1210/jc.2014-2850. 